\title{
A Comparative Study of Numerical Methods on Aerodynamic Characteristics of a Compressor Rotor at Near-stall Condition
}

\author{
Donghyun Kim*, Kuisoon Kim** and Jeongyeol Choi*** \\ Department of Aerospace Engineering, Pusan National University, Busan 609-735, Korea \\ Changmin Son**** \\ School of Mechanical Engineering, Pusan National University, Busan 609-735, Korea
}

\begin{abstract}
The present work performs three-dimensional flow calculations based on Reynolds Averaged Navier-Stokes (RANS) and Delayed Detached Eddy Simulation (DDES) to investigate the flow field of a transonic rotor (NASA Rotor 37) at near-stall condition. It is found that the DES approach is likely to predict well the complex flow characteristics such as secondary vortex or turbulent flow phenomenon than RANS approach, which is useful to describe the flow mechanism of a transonic compressor. Especially, the DES results show improvement of predicting the flow field in the wake region and the model captures reasonably well separated regions compared to the RANS model. Besides, it is discovered that the three-dimensional vortical flows after the vortex breakdown from the rotor tip region are widely distributed and its vortex structures are clearly present. Near the rotor leading edge, a part of the tip leakage flows in DES solution spill over into next passage of the blade owing to the separation vortex flow and the backflow is clearly seen around the trailing edge of rotor tip. Furthermore, the DES solution shows strong turbulent eddies especially in the rotor hub, rotor tip section and the downstream of rotor trailing edge compared to the RANS solution.
\end{abstract}

Key words: Transonic Compressor, Stall Inception, Detached Eddy Simulation, Secondary Vortex

\section{Introduction}

It is well known that flow characteristics of a transonic axial compressor is very complex due to tip leakage vortex, passage shock, and a lot of secondary flow structures within blade passages. Especially, they become more complicated as a compressor operating condition approaches low mass flow condition. In that condition, the secondary vortex structures and its interaction with passage shock can cause stall inception in a transonic compressor. In recent years, much attention has been paid to the flow phenomena of a transonic compressor at near-stall condition.

Suder [1] investigated a detailed experimental research to understand and quantify the development of blockage in the flow field of a transonic axial flow compressor rotor. It is observed that the overall blockage increases as the loading is increased from the high to low mass flow condition. Chima [2] performed three-dimensional calculations of the tip clearance flow in a transonic compressor. The results showed that a highly-rotational flow followed the shock-vortex interaction, with a large separated region at the operating point near stall. Hah et al. [3] studied the occurrence of short length scale rotating stall inception in a transonic compressor rotor using steady and unsteady flow simulations. They reported that the unsteadiness of the flow field is due to oscillation of tip clearance vortices and their interactions with passage shocks, suggesting it is an inherently unsteady phenomenon in a transonic compressor. Yamada et al. [4] identified vortex structures of a transonic axial compressor by unsteady simulations. It is indicated that the large blockage effect and
This is an Open Access article distributed under the terms of the Creative Commons Attribution Non-Commercial License (http://creativecommons.org/licenses/by$\mathrm{nc} / 3.0 /$ which permits unrestricted non-commercial use, distribution, and reproduction in any medium, provided the original work is properly cited.

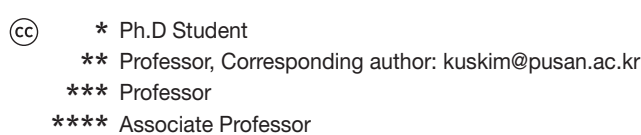


the unsteadiness due to the tip leakage vortex breakdown may be related to the spillage of the tip clearance flow at the stall inception. Wu et al. [5] conducted experimental and numerical investigations of the pre-stall behavior of an axial flow compressor rotor. They discovered that the cyclical motion of tip secondary vortex was the main source of the nearly periodic variation of efficiency. Cameron et al. [6] studied the role of tip leakage flow in axial compressor stall by the experiments and computations. The results confirmed that the compressor stalled when an interface between the approach flow and the tip-leakage flow reached the rotor leading edge plane as flow coefficient was reduced. $\mathrm{Hu}$ et al. [7] performed the steady and unsteady simulations of a transonic compressor rotor to examine the unsteadiness of the flow fields in the rotor. It is observed that the intensity of the tip clearance vortex, shock and interaction between them strengthens, which makes the interface between the incoming flow and tip clearance flow shift forward as the mass flow decreases. Shi et al. [8] numerically simulated the highly unsteady flow in an axial flow compressor at near-stall operating condition. The results explained the origin and the formation of the high entropy region in the compressor rotor near the blade leading edge, which can be closely related to the spike-type stall inception.

However, the flow mechanism of a compressor has not yet been fully investigated at near-stall condition and few studies have been conducted on comparisons of the flow structures using different numerical approaches. Considering the numerical results can be somewhat different depending on the capability of capturing secondary vortices, it is important to figure out the flow structures obtained from different numerical methods at near-stall condition. In the present work, the three-dimensional flow calculations based on Reynolds Averaged Navier-Stokes (RANS) and Delayed Detached Eddy Simulation (DDES) were performed to investigate the flow fields that lead to stall inception of a transonic axial compressor rotor at near-stall condition. To validate numerical methods, the both calculation results are compared with the experimental one.

\section{Compressor Model}

In the present work, the baseline configuration is a transonic axial compressor rotor, NASA Rotor 37 [9]. The isolated rotor was originally designed and tested as part of axial flow compressor stages at the NASA Lewis Research Center in the late 1970's by Reid and Moore [9]. The NASA Rotor 37 case was chosen as a "blind" test case for the ASME code assessment by the turbomachinery committee of ASME/IGTI in 1993 [10]. Fig. 1 shows the measurement locations of the rotor. Station 1 was located $4.19 \mathrm{~cm}$ upstream and station 4 was located $10.67 \mathrm{~cm}$ downstream of the blade hub leading edge. The design total pressure ratio is 2.106 at a measured mass flow of $20.19 \mathrm{~kg} / \mathrm{s}$. The rotor has 36 multiple circular-arc (MCA) blades with an aspect ratio of 1.19. The details of the compressor rotor are summarized in Table 1.

\section{Numerical Method}

\subsection{Numerical Scheme}

In the present study, the governing equations are the compressible Navier-Stokes equations. The governing equations were discretized numerically by a finite volume approach in space. Temporal integration used implicit second order accurate backward scheme to discretize a transient term in unsteady predictions and spatial integration used a second order accurate upwind differencing scheme. The meshes for RANS and DES were both identical in order to compare the flow characteristics at equivalent conditions. The result of transient calculations used a sampling data after 2 revolutions of a rotor for the unsteady time statistics.

For RANS approach, the Spalart-Allmaras (S-A)

Table 1. Specifications of the compressor

\begin{tabular}{|c|c|}
\hline Parameter & Value \\
\hline Blade number & 36 \\
\hline Hub radius at station 1 & $175.2 \mathrm{~mm}$ \\
\hline Tip radius at station 1 & $256.7 \mathrm{~mm}$ \\
\hline Aspect ratio & 1.19 \\
\hline Tip solidity & 1.29 \\
\hline \multirow{2}{*}{$\begin{array}{c}\text { Relative inlet Mach } \\
\text { number }\end{array}$} & Hub \\
\hline & Tip \\
\hline Tip clearance & $\begin{array}{c}0.356 \mathrm{~mm} \\
(0.45 \% \text { span })\end{array}$ \\
\hline Hub to tip ratio & 0.7 \\
\hline Design tip speed & $454 \mathrm{~m} / \mathrm{s}$ \\
\hline Rotating speed (100\%) & $\begin{array}{l}17188.7 \mathrm{rpm} \\
(1800 \mathrm{rad} / \mathrm{s})\end{array}$ \\
\hline Design total pressure ratio & 2.106 \\
\hline Design mass flow rate & $20.19 \mathrm{~kg} / \mathrm{s}$ \\
\hline Choking mass flow rate & $20.93 \mathrm{~kg} / \mathrm{s}$ \\
\hline
\end{tabular}

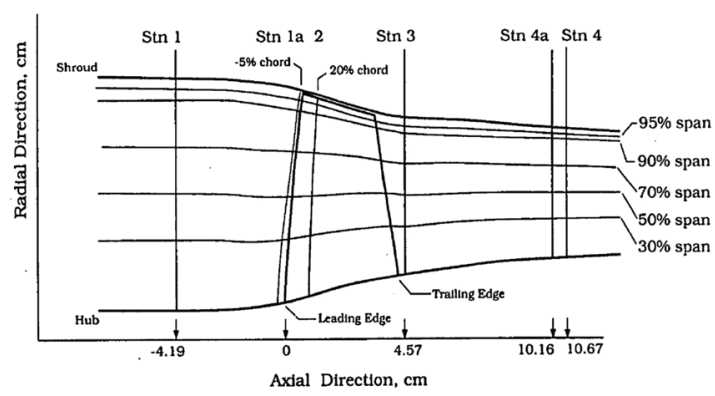

Fig. 1. Measurement stations [10] 
turbulence model was adopted. This one-equation model was designed to give reasonably good predictions of wake flows, flat-plate boundary layers and shows improvement in the prediction of flows with adverse pressure gradients [11]. To compare the predictive capability of unsteadiness against the RANS approach, the Detached Eddy Simulation (DES) method based on S-A model was used as a hybrid RANS/ LES turbulence model. Compared to classical LES methods, DES saves orders of magnitude of computing power for high Reynolds number flows, due to the moderate costs of the RANS model in the boundary layer region, but still offers some of the advantages of an LES method in separated regions [12]. The S-A based DES model was called "DES97" developed by Spalart et al. in 1997 [13]. In the present work, the Delayed DES (DDES) formulation based on the S-A model was employed [14]. This is a simple modification to DES97. The standard S-A model uses the distance to the closest wall as the definition for the length scale $d$, which plays a major role in determining the level of production and destruction of turbulent viscosity. In DES97 model, the length scale $d$ is replaced with a new length scale defined as [13]:

$$
\tilde{d}=\min \left(d, C_{D E S} \Delta\right)
$$

where the grid spacing $\Delta$ is $\max (\Delta x, \Delta y, \Delta z), C_{D E S}$ is the DES model constant. Using this algorithm, the model acts in a RANS mode when $d<<\Delta$ and in a LES mode when $d>>$. However, this model has incorrect behavior when the grid spacing is less than boundary layer thickness $\delta$. In the case of $\Delta<\delta$, the DES model can activate the LES mode inside the boundary layer, where the grid is not fine enough to sustain resolved turbulence [15]. Therefore, a new formulation of DES is suggested to preserve the RANS mode throughout the boundary layer. This is called DDES formulation written as follows [14]:

$$
\begin{aligned}
& \tilde{d} \equiv d-f_{d} \max \left(0, d-C_{D E S} \Delta\right) \\
& f_{d} \equiv 1-\tanh \left(\left(8 r_{d}\right)^{3}\right) \\
& r_{d} \equiv \frac{v_{t}+v}{\sqrt{U_{i, j} U_{i, j}} \kappa^{2} d^{2}} ; \kappa=0.41
\end{aligned}
$$

where $v_{t}$ is the kinematic eddy viscosity, $v$ the molecular viscosity, $U_{i, j}$ the velocity gradients, and the Karman constant, and the distance to the wall. The function $f_{d}$ is designed to be 1 in the LES region, where $r_{d} \ll<1$ or $r_{d}=0$. The function $r_{d}$ equals 1 in a logarithmic layer, and falls to 0 gradually towards the edge of the boundary layer. Therefore, setting $f_{d}$ to 0 yields RANS $(\tilde{d}=d)$, while setting it to 1 gives DES97 $\left(\tilde{d}=\min \left(d, C_{D E S} \Delta\right)\right)[14]$. The value of $C_{D E S}$ was determined to
0.2 as used in the literature [16]. The present work carries out the computations using commercial flow analysis program, ANSYS-Fluent.

\subsection{Computational Grid and Boundary Conditions}

The overall grid system used in the present work is shown in Fig. 2. An O-type grid near the blade surfaces, and a structured $\mathrm{H} / \mathrm{J} / \mathrm{C} / \mathrm{L}$-type grid in the other regions were generated. The grids around the inlet and outlet blocks consist of $20 \times 72 \times 154$ and $50 \times 80 \times 154$ grid points, respectively, while the O-type grids surrounding the blade walls are constructed with $280 \times 8 \times 154$ grid points. The total number of grids used in the calculation was determined to be approximately 3.1 million cells. Minimum grid spacing at the solid wall was less than $9 \times 10^{-5} \mathrm{~m}$. On the wall surface where the law of the wall was used to estimate the wall shear stresses, the normalized first grid distance normal to the wall, y+ was around 30. By using wall function model, the computational resource can be significantly reduced. For the computational domain, the total pressure, total temperature, and flow angle were specified at the inlet and average static pressure was imposed at the outlet. Besides, no-slip and adiabatic conditions were adopted for the solid walls and rotational periodicity was applied to lateral boundaries.

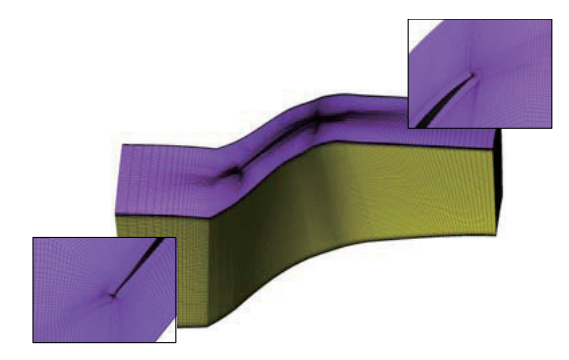

Fig. 2. Computational domain

\section{Results and Discussion}

\subsection{Validation}

To validate numerical methods used in this study, the computational results using the S-A RANS model and S-A based DES model are compared with experimental data at near-peak efficiency and at near-stall condition which are approximately $98 \%$ and $93 \%$ of choking mass flow condition, respectively. The radial distributions of total pressure, total temperature ratio were presented in Figs. 3 and 4. Those results are extracted from the pitch-wise mass averaged data. The uncertainties of experimental data are presented in Table 2. 
Table 2. Measurement uncertainty

\begin{tabular}{|c|c|}
\hline Parameter & Value \\
\hline \hline Massflow, kg/sec & \pm 0.3 \\
\hline Flow angle, deg. & \pm 1.0 \\
\hline Total temperature, $\mathrm{K}$ & \pm 0.6 \\
\hline Total pressure, $\mathrm{N} / \mathrm{cm}^{2}$ & \pm 0.01 \\
\hline
\end{tabular}

Figure 3 shows the total pressure and total temperature distributions at near-peak efficiency condition. For comparison, the RANS CFD results from reference [10] are also included. In Figs. 3 (a) and (b), there is a little difference between the CFD and experimental results, but the performance curves show a similar trend overall considering estimated uncertainty of measurement. Specifically, the temperature distribution of the DES solution near the rotor tip is slightly lower than experimental one but shows a good matching with measurement in the rest of regions compared to the RANS solution.

Figure 4 shows the present CFD results of total pressure and total temperature distributions at near-stall condition. In Fig. 4 (a), the overall trend of total pressure ratio using

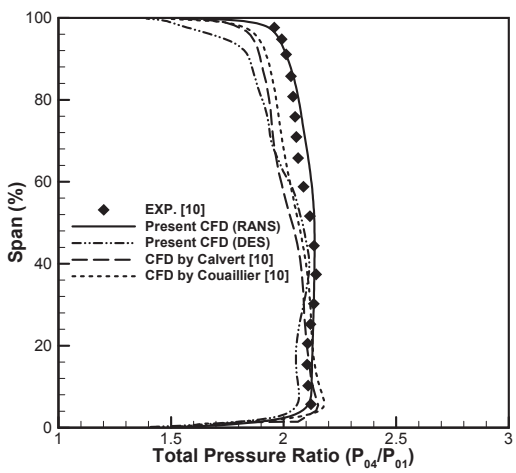

(a) Total pressure distribution

Fig. 3. Radial performances at near-peak efficiency condition

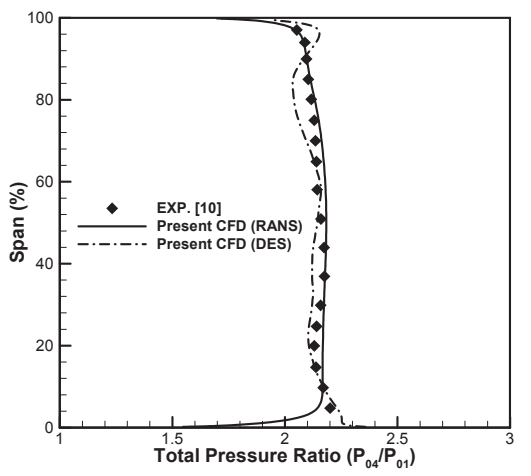

(a) Total pressure distribution both model is generally agreed with the experimental one. In addition, the DES solution near hub region is well matched with measurement than RANS solution. In Fig. 4 (b), the computed total temperature distribution is well agreed with the experimental data as well. Therefore, it is considered that the numerical method to investigate the flow field of the compressor is acceptable in the present study.

\subsection{Comparisons of Flow Characteristics}

Figure 5 shows the radial exit angle distributions at nearstall condition. The exit specified in this result is positioned at station 3 downstream of the rotor trailing edge. Those angles are calculated from the pitch-wise mass averaged velocity components. In common with the result of Figs. 3 and 4, the trends of both CFD results are similar to the experimental one. Especially, it is observed that the overall angle distribution of the DES solution is well agreed with experimental data than the RANS solution except for rotor tip region. This difference near the tip is due to the computation error by the use of wall function to calculate velocity profiles in the boundary layers.

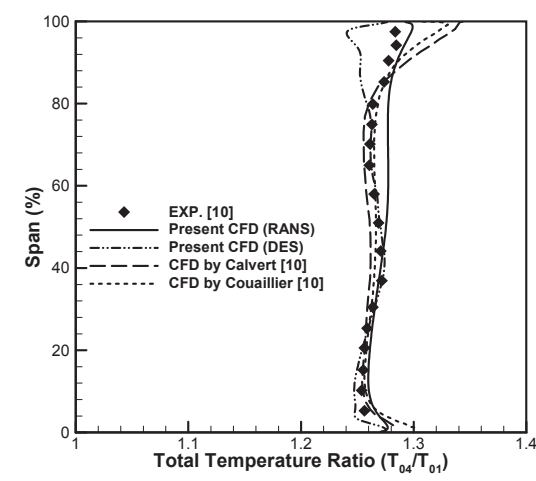

(b) Total temperature distribution

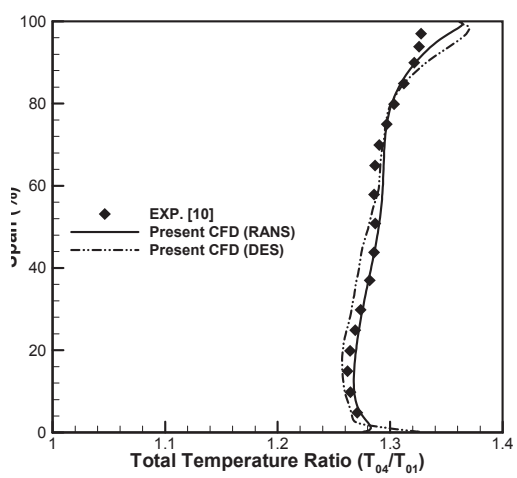

(b) Total temperature distribution

Fig. 4. Radial performances at near-stall condition 


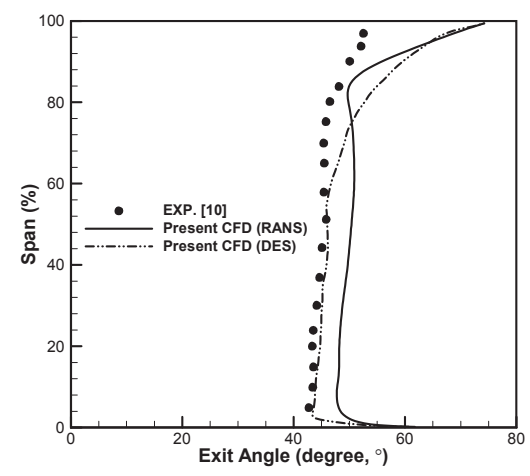

Fig. 5. Radial exit angle distributions at near-stall condition

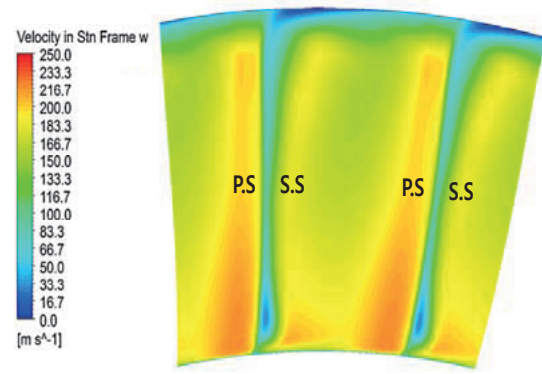

(a) RANS
Figure 6 shows the axial velocity distribution from $10 \%$ axial chord downstream of rotor trailing edge at near-stall condition. The RANS and time-averaged DES result is shown in the figure, respectively. As can be seen in Fig. 6, the axial velocity of RANS solution is less than the DES solution from hub to $70 \%$ span. The decrease in axial velocity components of RANS solution results in the increase of exit angle distribution.

Figure 7 shows the velocity streamlines near rotor hub suction surface at near-stall condition. In the RANS solution, the hub corner separation region is overestimated than the DES solution. This causes large total pressure drop while the

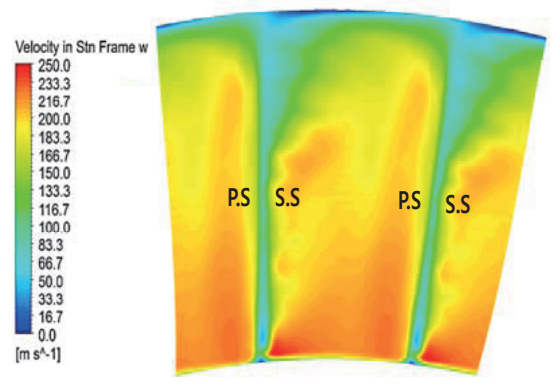

(b) DES (Time-averaged)

Fig. 6. Axial velocity distribution at near-stall condition ( $10 \%$ axial chord downstream of rotor TE)

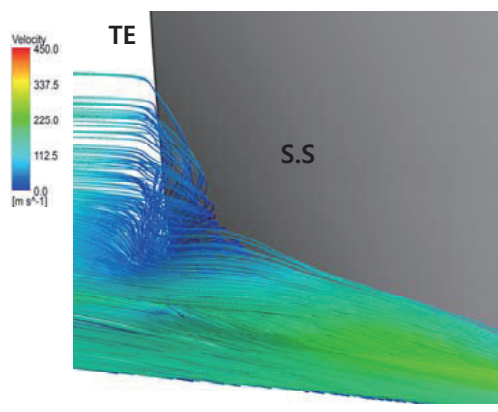

(a) RANS

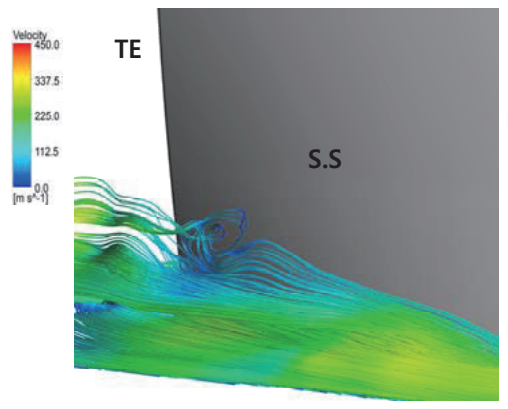

(b) DES

Fig. 7. Velocity streamlines near rotor hub suction surface at near-stall condition

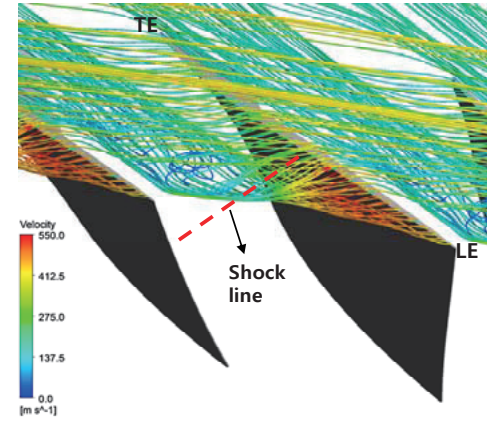

(a) RANS

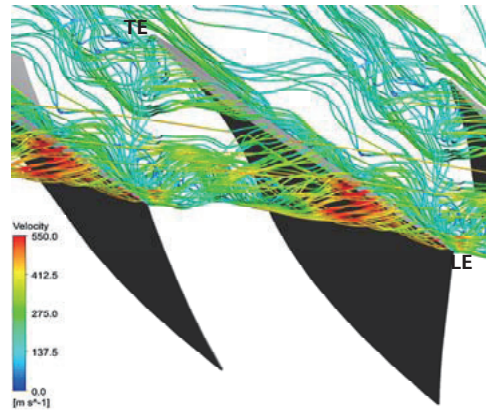

(b) DES

Fig. 8. Tip leakage flow pattern at near-stall condition 
DES model captures reasonably well the separated region.

Figure 8 shows the rotor tip leakage flows predicted by RANS and DES approach, respectively. The instantaneous DES result is presented. As seen in Fig. 8, it is observed that the tip leakage vortex passes from the upstream of rotor tip to the downstream of adjacent blade. Besides, the flow expansion in the tip leakage vortex downstream of the shock wave due to the strong adverse pressure gradient, which is called by vortex breakdown, occurs in the blade passage. In DES solution, it is discovered that the three-dimensional vortical flows from the rotor tip region are widely distributed and its vortex structures are well captured than the RANS solution.

Figure 9 shows the relative velocity vectors near the rotor blade surfaces at $98 \%$ span. As shown in Fig. 9 (a), the RANS and DES results indicate that the rotor tip clearance flow moves downstream of adjacent blade row, but a part of the tip leakage flows in DES solution spill over into next passage of the blade owing to the separation vortex as previously presented in Fig. 8 (b). Furthermore, there are different

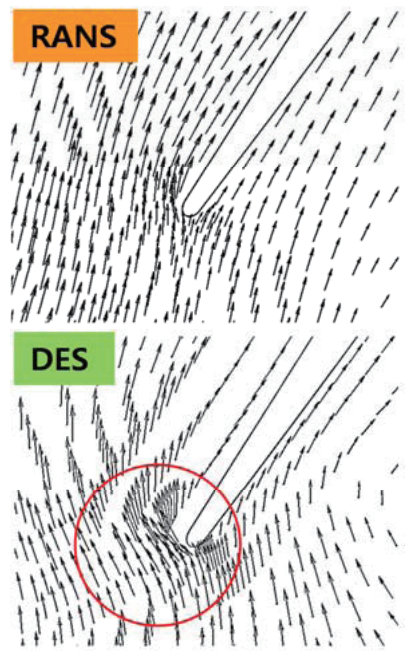

(a) Flow vectors around rotor leading edge

Fig. 9. Relative velocity vectors near blade surfaces ( $98 \%$ span)

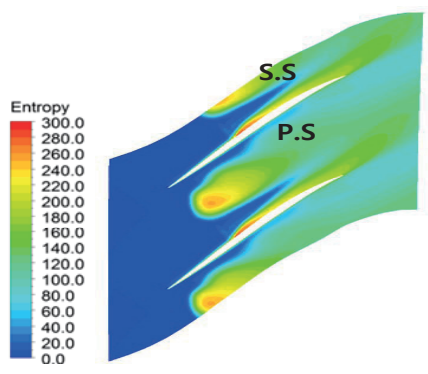

(a) RANS flow patterns between the RANS and DES solutions in Fig 9 (b). Near the rotor trailing edge, the flow separation on blade suction surface is observed and the backflow is clearly seen in DES solution. This is because the low energy fluids downstream of the separation vortex are driven upstream by the adverse pressure gradient [17].

Figure 10 shows the entropy distributions near the rotor tip surfaces at $95 \%$ span. The RANS and time-averaged DES result is shown in the figure, respectively. The entropy rise is defined as following:

$$
\Delta s=C_{p} \ln \left(\frac{T}{T_{r e f}}\right)-R \ln \left(\frac{p}{p_{r e f}}\right)
$$

In Figs. 10 (a) and (b), the sudden rise of entropy is observed in the blade passage due to the vortex breakdown and it is shown that the RANS solution has the higher maximum entropy region compared to the DES solution, but the distribution of high entropy region for the DES solution is wider than the RANS solution. For this reason, the rotor exit

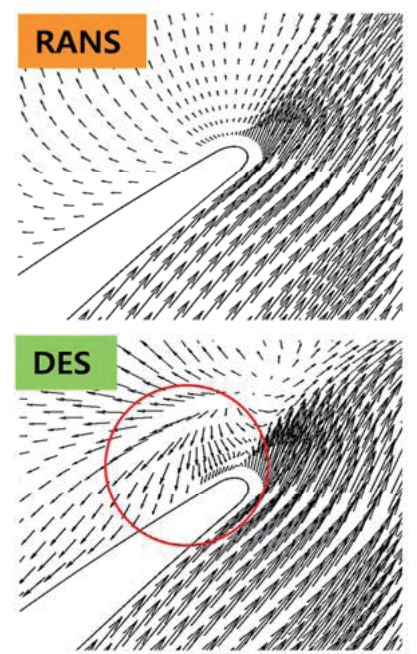

(b) Flow vectors around rotor trailing edge

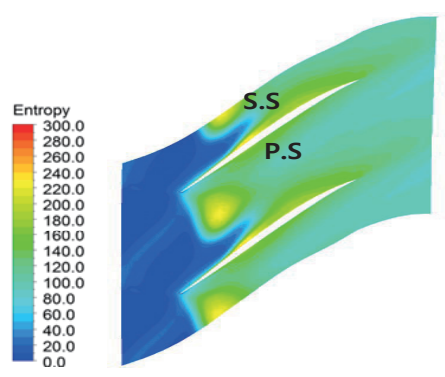

(b) DES (Time-averaged)

Fig. 10. Entropy distributions at rotor tip surface ( $95 \%$ span) 
entropy of DES solution is higher than the RANS solution. This phenomenon corresponds with the characteristics of total temperature distribution as mentioned in Fig. 4. Additionally, it seems that finer turbulent resolution using near wall treatment is needed to capture the smaller turbulent eddies in the tip region.

Figure 11 and Fig. 12 show the iso-surfaces of Q-criterion around rotor suction surfaces and the rotor limiting streamlines. The Q-criterion is used to identify the vortex cores and to visualize the turbulent eddy structures [18]. It means the averaged second invariant of the velocity gradient written as follows:

$$
Q=\frac{1}{2}\left(\Omega^{2}-S^{2}\right)
$$

where $\Omega$ is vorticity magnitude, $S$ is mean strain rate. It is found that the two vortex structures predicted by RANS and DES are clearly different from each other as seen in Figs. 11 and 12. The DES solution shows strong turbulent eddies with various sizes especially in the rotor hub and tip section and the downstream of rotor trailing edge compared to the RANS

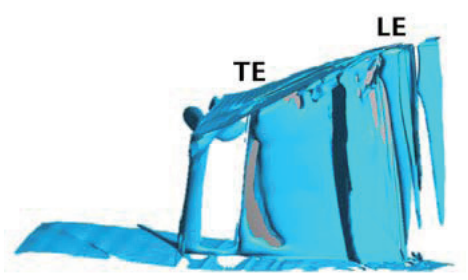

(a) RANS, iso-surface $Q=5 \times 10^{7} \mathrm{~s}^{-2}$

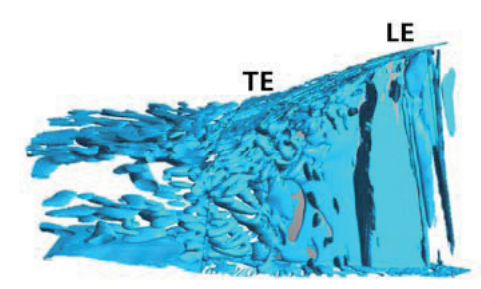

(b) DES, iso-surface $Q=5 \times 10^{7} \mathrm{~s}^{-2}$

Fig. 11. Iso Q-qriterion around rotor suction surface

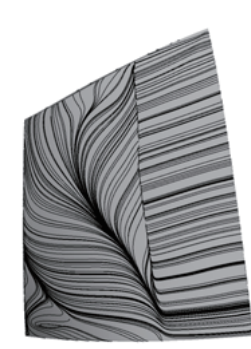

(a) RANS

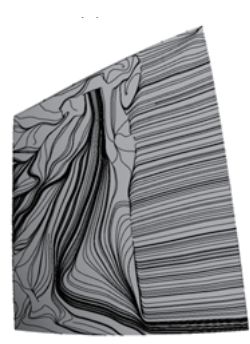

(b) DES
Fig. 12. Limiting streamlines on rotor suction surface solution. As for the rotor limiting streamlines, it is observed that the vortex flow region of RANS solution dominates in hub region. However, the vortical flow motions occur randomly in a wide range from hub to tip region for the DES solution.

\section{Conclusions}

The present work performs the three-dimensional flow calculations based on Reynolds Averaged Navier-Stokes (RANS) and Delayed Detached Eddy Simulation (DDES) to investigate the flow field of a transonic axial compressor rotor (NASA Rotor 37) at near-stall condition. It is found that the DES approach is likely to predict well the complex flow characteristics such as secondary vortex or turbulent flow phenomenon than RANS approach, which is useful to describe the flow mechanism of a transonic compressor at near-stall condition. The results are summarized as follows:

(1) The result of the DES model using wall function shows improvement of predicting the flow field in the wake region of the rotor and the model captures reasonably well separated regions compared to the RANS model. In the tip flow region, it seems that finer turbulent resolution using near wall treatment is needed to capture the smaller turbulent eddies.

(2) In DES solution, it is discovered that the threedimensional vortical flows after the vortex breakdown from the rotor tip region are widely distributed and its vortex structures are clearly present.

(3) There are several different flow patterns near the rotor blade surfaces between the RANS and DES solutions. Near the rotor leading edge, a part of the leakage flows in DES solution spill over into next passage of the blade owing to the separation vortex flow as shown in the tip leakage flow patterns. Furthermore, the flow separation on blade suction surface is observed and the backflow is clearly seen in DES solution near the trailing edge of rotor tip.

(4) It is found that the turbulent eddy structures predicted by RANS and DES are clearly different from each other. The DES result shows strong turbulent flow eddies especially in the rotor hub, rotor tip and the downstream of rotor trailing edge compared to the RANS solution.

\section{Acknowledgement}

This work was supported by the Human Resources in Energy Technology program of the Korea Institute of Energy Technology Evaluation and Planning (KETEP) grant funded by the Korea government Ministry of Trade, 
Industry and Energy (No.20144030200570). Also, this work was supported by the Human Resources Program in Energy Technology of the KETEP granted financial resource from the Ministry of Trade, Industry \& Energy, Republic of Korea (No.20124030200020).

\section{References}

[1] Suder, K. L., "Blockage Development in a Transonic, Axial Compressor Rotor", ASME Journal of Turbomachinery, Vol. 120, 1998, pp. 465-476.

[2] Chima, R. V., "Calculation of Tip Clearance Effects in a Transonic Compressor Rotor", ASME Journal of Turbomachinery, Vol. 120, 1998, pp. 131-140.

[3] Hah, C., Bergner, J. and Schiffer, H. P., "Short Length-Scale Rotating Stall Inception in a Transonic Axial Compressor - Criteria and Mechanisms", ASME Paper, No. GT2006-90045, 2006.

[4] Yamada, K., Funazaki, K. and Furukawa, M., "The Behavior of Tip Clearance Flow at Near-Stall Condition in a Transonic Axial Compressor Rotor", ASME Paper, No. GT2007-27725, 2007.

[5] Wu, Y., Li, Q., Tian, J. and Chu, W., "Investigation of Pre-Stall Behavior in an Axial Compressor Rotor-Part I: Unsteadiness of Tip Clearance Flow", ASME Journal of Turbomachinery, Vol. 134, 2012.

[6] Cameron, J. D., Bennington, M. A., Ross, M. H., Morris, S. C., Du, J., Lin, F. and Chen, J., "The Influence of Tip Clearance Momentum Flux on Stall Inception in a HighSpeed Axial Compressor", ASME Journal of Turbomachinery, Vol. 135, 2013.

[7] Hu, J. F., Zhu, X. C., Ou-Yang, H., Tian, Y. D., Wu, X. Q., Zhao, G. and Du Z. H., "The Unsteadiness of Tip Clearance Flow and its Effect to Stability of Transonic Axial Compressor", Journal of Theoretical and Applied Mechanics, Vol. 51, No. 2, 2013, pp. 431-438.
[8] Shi, K., Fu, S. and Morris, S. C., "IDDES Study of the Shock Induced Flow Separation in a Transonic Compressor Rotor at Near Stall Condition", ASME Paper, No. GT201427275, 2014.

[9] Reid, L. and Moore, R. D., "Design and Overall Performance of Four Highly Loaded, High-Speed Inlet Stages for an Advanced, High-Pressure-Ratio Core Compressor", NASA Technical Paper 1337, 1978.

[10] Dunham, J., "CFD Validation for Propulsion System Components", AGARD Advisory Report 355, 1998.

[11] Bardina, J. E., Huang, P. G. and Coakley, T. J., "Turbulence Modeling Validation, Testing, and Development", NASA Technical Memorandum 110446, 1997.

[12] Menter, F. R. and Kuntz, M., "Adaptation of EddyViscosity Turbulence Models to Unsteady Separated Flow Behind Vehicles", Proceedings of The Aerodynamics of Heavy Vehicles: Trucks, Busses and Trains, Asilomar, CA, 2002.

[13] Spalart, P. R., Jou, W-H., Strelets, M. and Allmaras, S. R., "Comments on the Feasibility of LES for Wings, and on a Hybrid RANS/LES Approach", Proceedings of the First AFOSR International Conference on DNS/LES, 1997.

[14] Spalart, P. R., Deck, S., Shur, M. L., Squires, K. D., Strelets, M. Kh. and Travin, A., "A new version of detachededdy simulation, resistant to ambiguous grid densities", Journal of Theoretical and Computational Fluid Dynamics, 2006.

[15] ANSYS Fluent Theory Guide, ANSYS, Inc., 2013.

[16] Gu, C., Chen, M., Li, X. and Feng, F., "Application of Delayed Detached Eddy Simulation and RANS to Compressor Cascade Flow", ASME Paper, No. GT2008-50040, 2008.

[17] Yamada, K., Kikuta, H., Iwakiri K., Furukawa, M. and Gunjishima, S., "An Explanation for Flow Features of SpikeType Stall Inception in an Axial Compressor Rotor", ASME Journal of Turbomachinery, Vol. 135, 2013.

[18] Jeong, J. and Hussain, F., "On the Identification of a Vortex”, Journal of Fluid Mechanism, Vol. 285, 1995, pp. 69-94. 